\title{
EULAR report on the use of ultrasonography in painful knee osteoarthritis. Part 1: Prevalence of inflammation in osteoarthritis
}

\author{
M A D'Agostino, P Conaghan, M Le Bars, G Baron, W Grassi, E Martin-Mola, \\ R Wakefield, J-L Brasseur, A So, M Backhaus, M Malaise, G Burmester, N Schmidely, \\ P Ravaud, M Dougados, P Emery
}

See end of article for authors' affiliations

\section{Correspondence to:} Professor M Dougados, Rheumatology

Department, Cochin Hospital, 27, rue du Faubourg St Jacques, 75014 Paris, France; maxime.dougados@ cch.ap-hop-paris.fr

Accepted 23 April 2005 Published Online First 5 May 2005

Objectives: To assess the prevalence of inflammation in subjects with chronic painful knee osteoarthritis (OA), as determined by the presence of synovitis or joint effusion at ultrasonography (US); and to evaluate the correlation between synovitis, effusion, and clinical parameters.

Methods: A cross sectional, multicentre, European study was conducted under the umbrella of EULARESCISIT. Subjects had primary chronic knee OA (ACR criteria) with pain during physical activity $\geqslant 30 \mathrm{~mm}$ for at least 48 hours. Clinical parameters were collected by a rheumatologist and an US examination of the painful knee was performed by a radiologist or rheumatologist within 72 hours of the clinical examination. Ultrasonographic synovitis was defined as synovial thickness $\geqslant 4 \mathrm{~mm}$ and diffuse or nodular appearance, and a joint effusion was defined as effusion depth $\geqslant 4 \mathrm{~mm}$.

Results: 600 patients with painful knee OA were analysed. At US 16 (2.7\%) had synovitis alone, 85 (14.2\%) had both synovitis and effusion, 177 (29.5\%) had joint effusion alone, and 322 (53.7\%) had no inflammation according to the definitions employed. Multivariate analysis showed that inflammation seen by US correlated statistically with advanced radiographic disease (Kellgren-Lawrence grade $\geqslant 3$; odds ratio $(O R)=2.20$ and 1.91 for synovitis and joint effusion, respectively), and with clinical signs and symptoms suggestive of an inflammatory "flare", such as joint effusion on clinical examination (OR = 1.97 and 2.70 for synovitis and joint effusion, respectively) or sudden aggravation of knee pain $(O R=1.77$ for joint effusion).

Conclusion: US can detect synovial inflammation and effusion in painful knee OA, which correlate significantly with knee synovitis, effusion, and clinical parameters suggestive of an inflammatory "flare".

$\mathrm{P}$ ain is the predominant feature of clinical knee osteoarthritis (OA), but its origin is not clearly established. ${ }^{12}$ Among the different tissues contributing to the sources of pain, synovial tissue or subchondral bone, or both, may have an important role. ${ }^{3-6}$ It has been assumed that pain and functional impairment associated with a clinical "flare" of $\mathrm{OA}$ are also associated with inflammation of synovial tissue. Several arthroscopy studies have shown that synovitis is common in painful knee OA. ${ }^{7-10}$ Magnetic resonance imaging (MRI) has been validated in the detection of synovitis in $\mathrm{OA}_{,}{ }^{11}$ and an association between pain and synovial thickening and effusions detected by MRI has been reported. ${ }^{12}$

A link between synovial inflammation and progression of structural damage has been suggested by a number of studies. A 1 year longitudinal study demonstrated that a larger volume of effusion aspirations for painful OA knee predicted OA progression as defined by increased joint space narrowing. ${ }^{10}$ Arthroscopic studies have suggested that baseline synovitis scores predict subsequent worsening of chondropathy in post-traumatic patellofemoral damage ${ }^{10}$ and in a large cohort of patients with OA knee. ${ }^{7}$

The clinical diagnosis of synovial inflammation in knee OA is empirical and based on the presence of symptoms such as a sudden change in the level of pain, pain at night, prolonged morning stiffness, and the presence of an effusion on clinical examination. However, there are no validated clinical algorithms available. ${ }^{3}{ }^{4}$ Indeed there are limited data from large cohorts on the prevalence of synovial hypertrophy and

effusions in knee OA, making it difficult to understand their true association with knee symptoms and examination findings.

A more objective assessment of inflammatory findings in OA might be obtained by imaging techniques such as arthroscopy, MRI, or ultrasonography (US). ${ }^{13}$ US is noninvasive and is more easily used in the evaluation of a large cohort than other imaging modalities. Currently, US seems more useful in the evaluation of joint effusion and synovitis (both hypertrophy and morphology) ${ }^{14-17}$ than in cartilage examination (although cartilage assessment may be obtained, especially in the patellofemoral compartment). ${ }^{15} 16$ It has been demonstrated that US is a valid and reliable instrument for the assessment of synovial disease, with results comparable to those obtained by MRI or arthroscopy, or both. ${ }^{14} 1819$

US features were used as the "gold standard", and this study aimed at assessing the prevalence of inflammation, as defined by the presence of synovitis and effusion, in subjects with chronic primary, painful, knee OA. We also evaluated the correlation between US synovitis and joint effusion, and between these two variables and clinical features.

Abbreviations: K\&L, Kellgren and Lawrence; MRI, magnetic resonance imaging; OA, osteoarthritis; US, ultrasonography; VAS, visual analogue scale; WOMAC, Western Ontario and McMaster Universities Osteoarthritis Index 


\section{METHODS}

\section{Study design}

This was a cross sectional, multicentre, prospective European study conducted under the umbrella of the European League Against Rheumatism (EULAR) Standing Committee for International Clinical Studies Including Therapeutics Trials (ESCISIT). Appropriate ethics committee permission was obtained in each country, and written informed consent was obtained from every patient before study participation.

Outpatients with chronic, painful, primary knee OA from seven European countries (Belgium, France, Germany, Italy, Spain, Switzerland, and the United Kingdom) were recruited by 50 rheumatologists who performed the clinical examination. A total of 29 investigators (radiologists or rheumatologists) performed the US examination of the knees for each of the subjects included. Selection of these investigators was based on their experience with musculoskeletal US.

Subjects were examined only once by each of the investigators within a maximum 72 hour time interval. To minimise the risk of bias, the investigator who performed the US examination was unaware of the clinical results before performing the examination, but the ultrasonographer was able to communicate in writing the results of the US examination to the clinical investigator. Data were recorded by the investigators on two separate case report forms, and forwarded to a central data entry and quality control service.

The main inclusion criteria were men or women over 18 years of age with primary knee OA according to the American College of Rheumatology ${ }^{20}$; with first OA symptoms detected at least 6 months before study entry; with radiographic evidence of $\mathrm{OA}$ for the studied knee defined by the Kellgren and Lawrence $(\mathrm{K} \& \mathrm{~L})$ grade $1-4^{2122}$; and with a pain intensity during physical activities in the previous 48 hours $\geqslant 30 \mathrm{~mm}$ measured on a $100 \mathrm{~mm}$ visual analogue scale (VAS).

Exclusion criteria included disabling OA and/or subjects with grade 4 Steinbrocker functional score ${ }^{23}$; secondary OA as defined by the Group for the Respect of Ethics and Excellence in Science (GREES) ${ }^{5}$; a history of injury to the studied knee in the 6 months before study entry; joint replacement (partial or total) or osteotomy of the studied joint; history of arthroscopy of the studied joint within the previous year; and an intra-articular injection of steroids given during the previous 4 weeks and/or an intra-articular injection of radionuclide given in the 3 months before the start of the study.

\section{US variables}

The sensitivity of power Doppler in detecting synovial inflammation is highly machine dependent, ${ }^{24}$ and therefore we decided to evaluate the synovial membrane using B mode US only.

At the time of devising this study, no widely agreed, standardised definitions of US pathological findings, or widely recommended acquisition protocols, were available. On US examination, the normal synovial membrane of the knee appears as a thin echoic layer up to $3 \mathrm{~mm}$ thick. Using B mode US, two main morphological patterns of synovial inflammation have been described: the first consists of hypoechoic tissue which is uniformly distributed in the joint cavity (diffuse appearance), whereas the second also has a hypoechoic appearance but exhibits a villous pattern (nodular appearance). ${ }^{13} 1819$ In studies in which power Doppler has been used to confirm the presence of vascularisation in the inflamed synovium, these two patterns were consistently found..$^{131819}$ We therefore produced definitions of synovitis and effusion based on the limited available published reports $^{13} 1819$ and included descriptions of synovial morphology. We employed a conservative "cut off point" for pathological dimensions reflecting synovial hypertrophy and effusion. Recommendations for acquisition were also based on previous reports..$^{25} 26$

Synovitis and effusion were measured and recorded only in the suprapatellar recess using US equipment with a high frequency linear array (minimum $10 \mathrm{MHz}$ ). The maximal synovial thickness and effusion depth were measured in millimetres using the longitudinal axis.

Synovitis was defined as hypoechoic synovial hypertrophy with thickness $\geqslant 4 \mathrm{~mm}$ and diffuse or nodular appearance, with the knee semiflexed at $45^{\circ}$, on the median longitudinal plane crossing the quadriceps tendon. This definition was used as the primary outcome. For this study and the remainder of this paper, the term "synovitis" refers to the presence of a hypoechoic, hypertrophic synovium. The presence of a hyperechoic area (for example, fibrous synovitis) in the joint space was not recorded. It was also decided not to regard synovial hypertrophy with a normal (as distinct from hypoechoic) appearance as synovitis, regardless of the thickness. The morphology of inflammatory synovitis was evaluated on a three point categorical scale as absent, nodular, or diffuse.

Joint effusion was defined as an anechoic area and was measured in the suprapatella recess, with the leg in full extension, and measured at the maximal depth observed with a longitudinal scan. It was recorded as absent if the effusion depth was $<4 \mathrm{~mm}$, and present if $\geqslant 4 \mathrm{~mm}$; this definition was used as the key secondary outcome.

To standardise the techniques and ensure the highest possible reproducibility, we performed a l day training session for the ultrasonographers before the start of the study. The object of this session was to standardise the US examination (transducer orientation, documentation, positioning, and adherence to standard planes) with respect to the recommended acquisition protocol and pathological definitions. At this training session, all ultrasonographers separately examined two subjects with painful knee OA.

\section{Clinical parameters}

The clinical parameters collected were demographic data, knee OA characteristics such as disease duration, and OA localisation (medial femorotibial, lateral femorotibial, or femoropatellar) and radiological severity based on the K\&L grade for each compartment. ${ }^{22}{ }^{23}$ The clinical severity of the studied knee during the previous 48 hours was also recorded. This included the patient's global assessment of knee pain using a $100 \mathrm{~mm} \mathrm{VAS,}{ }^{27}$ the patient's global assessment of clinical knee OA severity (100 mm VAS), ${ }^{27}$ the physician's global assessment of clinical knee OA severity (100 mm VAS), ${ }^{27}$ the Western Ontario and McMaster Universities Osteoarthritis Index (WOMAC) pain subscale (five items and overall score (100 mm VAS)), ${ }^{28}$ the WOMAC stiffness subscale (two items and overall score $(100 \mathrm{~mm}$ VAS $)),{ }^{28}$ the WOMAC physical function subscale (17 items and overall

\begin{tabular}{lll}
$\begin{array}{l}\text { Table } 1 \\
\text { subjects with painful knee OA, by country }\end{array}$ & $\begin{array}{l}\text { Number (\%) of enrolled and analysed } \\
\text { Enrolled } \\
\text { subjects } \\
\text { (n=643) }\end{array}$ & $\begin{array}{l}\text { Analysed } \\
\text { subjects } \\
\text { ( } \mathbf{n = 6 0 0 )}\end{array}$ \\
\hline Belgium & $26(4.0)$ & $26(4.3)$ \\
France & $250(38.9)$ & $224(37.3)$ \\
Germany & $35(5.4)$ & $30(5.0)$ \\
Italy & $102(15.9)$ & $102(17.0)$ \\
Spain & $83(12.9)$ & $80(13.3)$ \\
Switzerland & $68(10.6)$ & $62(10.3)$ \\
United Kingdom & $79(12.3)$ & $76(12.7)$ \\
\hline \multicolumn{3}{l}{} \\
\hline
\end{tabular}


score (100 mm VAS $)),{ }^{28}$ the duration of morning stiffness in minutes ${ }^{26}$; awakenings at night due to knee pain ${ }^{29}{ }^{30}$ using a four point verbal scale (no discomfort/no pain; minimal discomfort, intermittent pain/numbness; severe discomfort, intermittent or continuous pain resulting in awakening at night; very severe discomfort, continuous pain preventing sleep); sudden aggravation of knee pain in the previous 2 weeks on a five point verbal scale (not at all, minimal, moderate, severe, very severe); and knee joint effusion at clinical examination, on a four point verbal scale (not at all, minimal, moderate, important). We also recorded the previous (during the past 4 weeks) and concomitant symptomatic treatments for knee OA.

\section{Statistical analysis}

The definitions of confirmed inflammation, according to the cut off points mentioned above, were used to explore the relationship between synovitis and/or effusion at US and clinical parameters.

All collected clinical data were analysed as raw data, or transformed into binary variables. A composite index of clinical inflammation was calculated as the presence of at least two of the following variables: night awakening due to knee pain, prolonged morning stiffness ( $\geqslant 30$ minutes), severe or very severe/sudden aggravation of knee pain in the previous 2 weeks; and moderate or important joint effusion at clinical examination. ${ }^{27}$ For the WOMAC, derived variables were calculated. For each subscale, the total score was calculated as the sum of corresponding items (rated on a VAS). The normalised WOMAC score was calculated based on a weighted sum of subscale scores. The following weights were used: 0.20 for the pain subscale, 0.50 for the stiffness subscale, and 0.059 for the physical function subscale. ${ }^{27}$ For continuous data, a one way analysis of variance was used to

Table 2 Demographic and clinical characteristics of subjects with painful knee $\mathrm{OA}$ at baseline

\begin{tabular}{lll}
\hline Characteristics & $\begin{array}{l}\text { Subjects } \\
\text { (n=600) }\end{array}$ & p Value† \\
\hline Age (years) & $66.7(9.8)$ & 0.0125 \\
Body mass index $\left(\mathrm{kg} / \mathrm{m}^{2}\right)$ & $29.9(5.3)$ & 0.0001 \\
Sex & & 0.0001 \\
$\quad$ Male, No (\%) & $165(27.5)$ & \\
$\quad$ Female, No (\%) & $435(72.5)$ & \\
Disease duration (years) & $6.0(6.8)$ & 0.1029 \\
K\&L radiological grade $\geqslant 3^{*}$, No (\%) & $399(66.5)$ & 0.0063 \\
Knee pain intensity on VAS & $63(18.5)$ & 0.0001 \\
Joint effusion at clinical examinationt, No (\%) & $204(34.0)$ & 0.0001 \\
Sudden aggravation of knee pain in previous & $107(17.8)$ & 0.0001 \\
2 weeks, No (\%) & & \\
Duration of morning stiffness (min): & & \\
$\quad$ on VAS & $13.9(17.9)$ & 0.5603 \\
$\quad \geqslant 30$ minutest, No (\%) & $100(16.7)$ & 0.9571 \\
Night awakenings due to knee pain, No (\%) & $171(28.5)$ & 0.0438 \\
WOMAC§ & & \\
$\quad$ Pain subscale (0-100) & $51.7(20.2)$ & 0.0001 \\
$\quad$ Stiffness subscale (0-100) & $53.4(25.3)$ & 0.0001 \\
$\quad$ Physical function subscale (0-100) & $52.0(19.9)$ & 0.0001 \\
Clinical knee OA severity on VAS (0-100) & & \\
$\quad$ Patient's global assessment & $64.2(21.4)$ & 0.0001 \\
Physician's global assessment & $55.8(19.5)$ & 0.0001 \\
\hline
\end{tabular}

Results are shown as mean (SD) unless indicated otherwise. *K\&L, Kellgren and Lawrence radiological grade $\geqslant 3$ observed in both femorotibial compartments and/or in femoropatellar compartment of the knee studied; tjoint effusion was considered as a binary variable: "present" if graded moderate or important, "absent" if graded absent or minimal; only those subjects with effusion present are included here. fmorning stiffness was also considered as a binary variable: yes, if $\geqslant 30$ minutes and no, if $\leqslant 30$ minutes; §WOMAC, Western Ontario and McMaster Universities OA Index, Fisher's exact test for statistically significant differences between countries.

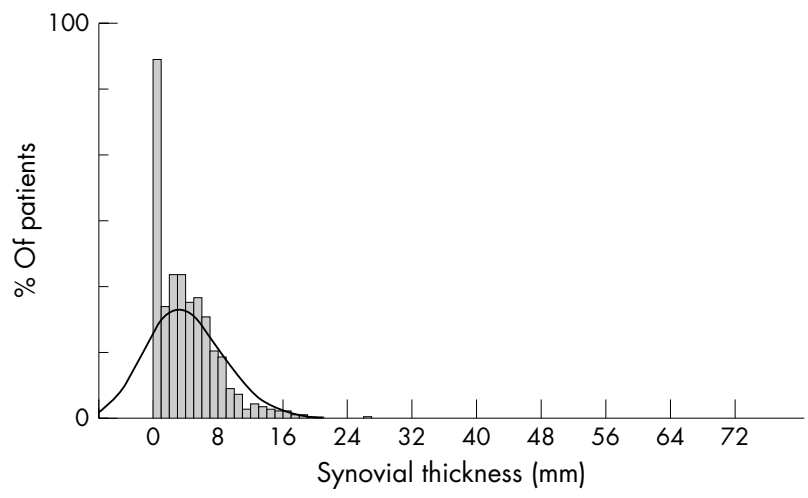

Figure 1 Synovitis at ultrasonography in subjects with painful knee OA: distribution of synovial thickness.

make comparisons between groups. For categorical data, a $\chi^{2}$ test (Fisher's exact test, when appropriate) was used.

For nominal data, we used the $\chi^{2}$ test with continuity corrections; for ordinal variables, the Mann-Whitney U test; and for continuous variables, Student's unpaired $t$ test. Pooled or separate variance estimates were used as appropriate. All specified analyses were planned to be performed at the $5 \%$ significance level using a two tailed alternative.

The prevalence of knee synovitis and joint effusion was calculated together with their $95 \%$ confidence intervals. To evaluate the correlation between the presence of knee synovitis, joint effusion, and clinical parameters of knee OA, univariate analyses were used to determine the strength of association between each variable and the primary outcome and to aid selection of the best variables for the multivariate analyses.

The appropriate univariate techniques were chosen according to the type of data. Those variables found to be strongly associated with the outcome measure $(p<0.20)$ were integrated in multivariate analyses. Building of the logistic regression model proceeded with stepwise selection until no variables met the criteria for entry $(p<0.05)$ or removal $(p>0.05)$, for the significance levels of the likelihood ratio test. All models were adjusted for a country effect. The same methodology was repeated for joint effusion. Clinical

Table 3 Prevalence of knee synovitis on ultrasonography in subjects with painful knee OA

\begin{tabular}{|c|c|c|c|}
\hline & \multicolumn{2}{|c|}{$\begin{array}{l}\text { Ultrasonographic } \\
\text { knee synovitis* }\end{array}$} & \multirow[b]{2}{*}{ p Valuet } \\
\hline & Absent & Present & \\
\hline Total number of subjects, No (\%) & $500(83.3)$ & $100(16.7)$ & \\
\hline $\begin{array}{l}\text { Synovial thickness }(\mathrm{mm}) \\
\text { Mean (SD) } \\
\text { Median (range) }\end{array}$ & $\begin{array}{l}1.3(1.5) \\
1(0-19)\end{array}$ & $\begin{array}{l}5.8(3.0) \\
5(4-29)\end{array}$ & $<0.0001$ \\
\hline $\begin{array}{l}\text { Appearance of synovial tissue, } \\
\text { No (\%) } \\
\text { Absent } \\
\text { Nodular } \\
\text { Diffuse }\end{array}$ & $\begin{array}{l}278(55.6) \\
75(15.0) \\
147(29.4)\end{array}$ & $\begin{array}{l}0(0) \\
56(56.0) \\
44(44.0)\end{array}$ & $<0.0001$ \\
\hline $\begin{array}{l}\text { Maximal effusion depth (mm) } \\
\text { Mean (SD) } \\
\text { Median (range) }\end{array}$ & $\begin{array}{l}3.4(4.5) \\
2.7(0-20)\end{array}$ & $\begin{array}{l}6.9(3.9) \\
6.1(0-71)\end{array}$ & $<0.0001$ \\
\hline
\end{tabular}

"Knee synovitis was defined as "present" if synovial thickness was $\geqslant 4 \mathrm{~mm}$ with diffuse or nodular appearance. tp Values refer to comparisons between subgroups with or without synovitis (Student's $t$ test for quantitative variables and $\chi^{2}$ test for qualitative variables). 
parameters were compared between countries using the appropriate statistical test. A similar exploratory analysis was then performed in subgroups of subjects with higher and advanced radiographic disease (K\&L grade $\geqslant 3$ ) or with an earlier radiographic stage $(K \& L$ grade $<3$ ), based on their predictive factors.

For the primary objective, a sample size of 600 subjects was required to achieve a precision of $\pm 2.5-4 \%$ for an observed prevalence ranging between $10 \%$ and $30 \%$. With respect to the secondary objectives, with this sample size, we also had $80 \%$ power to detect differences of $10-20 \%$ in the proportions of various clinical characteristics, with an observed prevalence ranging between $15 \%$ and $30 \%{ }^{31}$

\section{RESULTS}

Demographic and other baseline characteristics

Of 643 subjects enrolled in the study, 627 were potentially analysable; $16(2.5 \%)$ subjects had only one case report form available from clinical or US evaluation. Among these 627 subjects, $27(4.3 \%)$ subjects had a major protocol deviation, leaving 600 subjects who were included in the final analysis. Table 1 shows the distribution of subjects by country. Table 2 summarises the main baseline characteristics of the 600 analysed subjects. Some statistically significant differences in the baseline demographic and clinical data between countries were found (table 2).

Moderate or important knee joint effusion on clinical examination was present in $34.0 \%$ of subjects. When the inflammation composite score was considered, only $37.3 \%$ had at least two of the four clinical features listed above (expressed as binary variables). Of the 600 subjects, 410 $(68.3 \%)$ had recently used or were currently using drugs for their OA. The different classes of treatments were nonselective non-steroidal anti-inflammatory drugs in $34.8 \%$ of subjects, selective inhibitors of cyclo-oxygenase-2 in $17.1 \%$, paracetamol in $23.9 \%$, opioids in $15.6 \%$, and symptomatic slow acting drugs in $8.5 \%$.

\section{Prevalence of ultrasonographic knee synovitis}

In the total group the mean (SD) synovial thickness measured was $2.1(2.5) \mathrm{mm}$, and the median was $1.5 \mathrm{~mm}$ (range 0-29 mm). This distribution was not normal. The appearance of synovial tissue was recorded as absent, nodular, or diffuse in $46.3 \%, 21.8 \%$, and $31.8 \%$ of subjects, respectively $(p<0.0001)$. Figure 1 shows the distribution of synovial thickness in the overall group. Table 3 shows the distribution of synovial thickness and appearance as well as the maximal effusion depth in subjects with or without synovitis.

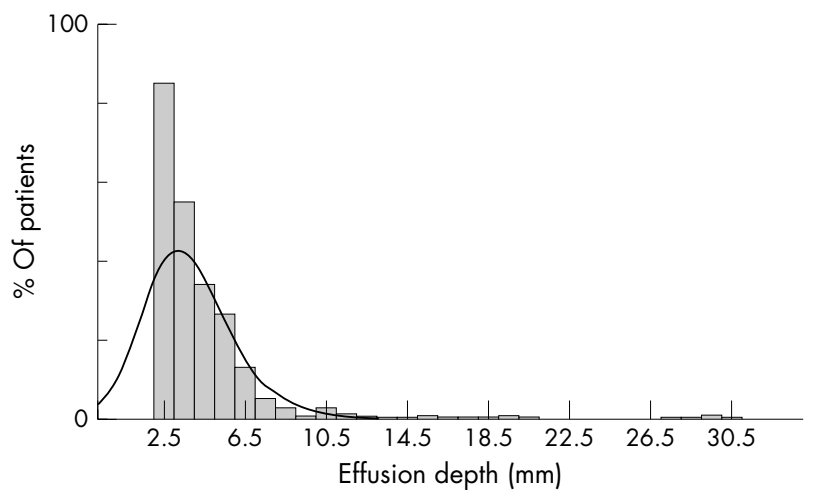

Figure 2 Effusion depth on ultrasonography in subjects with painful knee OA: distribution of effusion depth.
Table 4 Prevalence of joint effusion on ultrasonography in subjects with painful knee osteoarthritis

\begin{tabular}{|c|c|c|c|}
\hline & \multicolumn{2}{|c|}{$\begin{array}{l}\text { Ultrasonographic joint } \\
\text { effusion** }\end{array}$} & \multirow[b]{2}{*}{ p Value } \\
\hline & Absent & Present & \\
\hline Subjects, No (\%)‡ & $338(56.4)$ & $261(43.6)$ & \\
\hline Maximal effusion depth (mm) & & & $<0.0001$ \\
\hline Mean (SD) & $1.3(1.4)$ & $7.5(5)$ & \\
\hline Median (range) & $1.2(0-4)$ & $6.3(4-71)$ & \\
\hline Synovial thickness (mm) & & & $<0.0001$ \\
\hline Mean (SD) & $1.1(1.5)$ & $3.3(2.9)$ & \\
\hline Median (range) & $0.6(0-10.2)$ & $2.9(0-29)$ & \\
\hline $\begin{array}{l}\text { Appearance of synovial tissue, } \\
\text { No (\%) }\end{array}$ & & & $<0.0001$ \\
\hline Normal & $231(68.3)$ & $47(18.0)$ & \\
\hline Nodular & $34(10.1)$ & 97 (37.2) & \\
\hline Diffuse & $73(21.6)$ & $117(44.8)$ & \\
\hline
\end{tabular}

*Joint effusion was defined as present if depth $\geqslant 4 \mathrm{~mm}$; tp values refer to comparisons between subgroups with or without joint effusion. (Student's $t$ test for quantitative variables and $\chi^{2}$ test for qualitative variables); łmissing data for one patient.

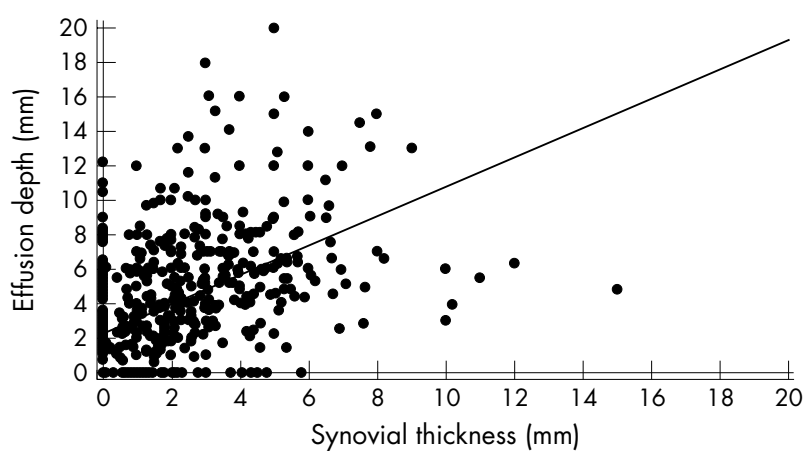

Figure 3 Relationship between ultrasonography synovial thickness and effusion depth using linear regression.

\section{Prevalence of ultrasonographic joint effusions}

The mean (SD) effusion depth measured in the total group was 4.0 (4.6) $\mathrm{mm}$, and the median was $3.2 \mathrm{~mm}$ (range 0$71 \mathrm{~mm})$. The distribution of the effusion depth was not normal.

Figure 2 shows the distribution of effusion depth in the overall group of patients. Table 4 shows the distribution of synovial thickness, synovial appearance, and the maximal effusion depth in subjects with or without effusion.

\section{Relationship between ultrasonographic knee synovitis and joint effusion}

Among subjects with painful knee OA, 322 (53.7\%) had neither US synovitis nor effusion, 177 (29.5\%) had effusion alone, 85 (14.2\%) had both synovitis and effusion, and only $16(2.7 \%)$ had synovitis alone. The association between knee synovitis and joint effusion was highly significant $(p<0.0001)$. Additionally, a strong relationship between synovial thickness and effusion depth was demonstrated using actual measurements rather than the predefined protocol cut off points (correlation coefficient $=0.51$, $\mathrm{p}<0.0001$ ) (fig 3).

As reported above, $37.3 \%$ of subjects had at least two of the four clinical features suggesting an inflammatory episode. In this subgroup, only $52.5 \%$ subjects had synovitis or effusion, or both, at US. 

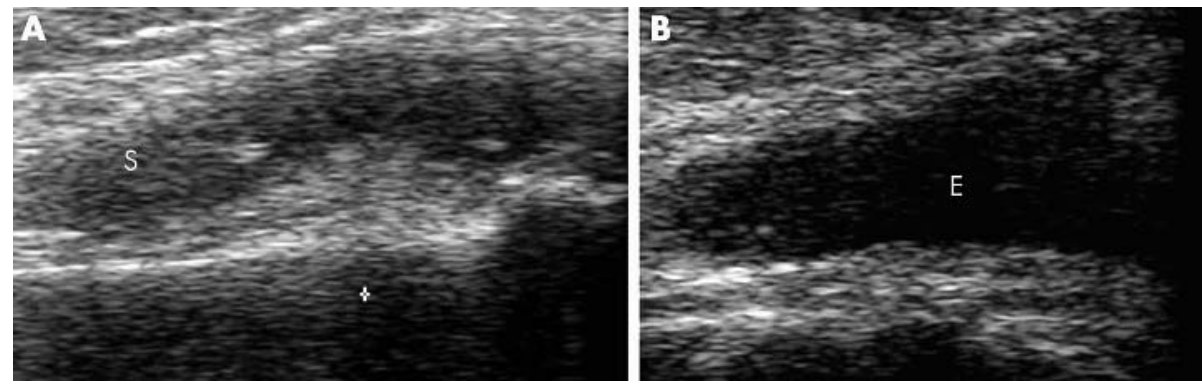

Figure 4 Synovitis (A) and joint effusion (B) on US in subjects with painful knee OA. (A) Synovitis in a longitudinal scan (suprapatellar recess). (B) Joint effusion in a longitudinal scan (suprapatellar recess): $S$, synovitis; $E$, effusion.

Figure 4 shows an example of synovitis (A) and joint effusion (B) detected by US.

\section{Univariate analysis}

Synovitis

For synovitis, the significant variables $(p<0.20)$ arising from the univariate analysis were $\operatorname{sex}(p=0.1772)$, weight $(\mathrm{p}=0.1649)$, height $(\mathrm{p}=0.1690), \mathrm{K} \& \mathrm{~L}$ radiological grade $\geqslant 3$ observed in both femorotibial compartments $(p<0.0001)$ or radiological grade $\geqslant 3$ observed in all three compartments of the studied knee $(p=0.0017)$, physician's global assessment of clinical knee OA severity $(p=0.0055)$, morning stiffness $\geqslant 30$ minutes $(p=0.0542)$, knee joint effusion at clinical examination $(\mathrm{p}<0.0001)$, and sudden aggravation of knee pain in the previous 2 weeks $(p=0.0479)$.

\section{Joint effusion}

For joint effusion, the significant variables $(p<0.20)$ arising from the univariate analysis were sex $(p=0.0016)$, weight $(p=0.1943)$, height $(p=0.0400)$, spine osteoarthritis $(p=0.0242), K \& L$ radiological grade $\geqslant 3$ observed in both femorotibial compartments of the studied knee $(\mathrm{p}<0.0001)$ and $K \& L$ radiological grade $\geqslant 3$ observed in all three compartments of the studied knee $(\mathrm{p}=0.0002)$, physician's global assessment of clinical knee OA severity $(p=0.0072)$, knee joint effusion at clinical examination $(\mathrm{p}<0.0001)$, sudden aggravation of knee pain in the previous 2 weeks $(\mathrm{p}=0.0441)$, and the composite index of inflammation $(\mathrm{p}=0.0024)$. The variables strongly associated with the outcome measures were integrated in the multivariate analysis.

Table 5 Relationship between knee synovitis or joint effusion on US and predictive features in subjects with painful knee OA. Summary of multivariate analyses-odds ratio estimates (adjusted by country)

\begin{tabular}{|c|c|c|c|c|}
\hline \multirow[b]{2}{*}{ Predictive factor } & \multicolumn{2}{|c|}{ US knee synovitis } & \multirow[b]{2}{*}{ p Value* } & \multirow[b]{2}{*}{ OR $(95 \% \mathrm{Cl}) \dagger$} \\
\hline & $\begin{array}{l}\text { Absent } \\
\text { No (\%) }\end{array}$ & $\begin{array}{l}\text { Present } \\
\text { No (\%) }\end{array}$ & & \\
\hline \multicolumn{5}{|l|}{ Radiological K\&L grades $\ddagger$} \\
\hline $3-4$ & $260(77.8)$ & $74(22.2)$ & $<0.0001$ & 2.20 (1.33 to 3.64$)$ \\
\hline $0-2$ & $240(90.2)$ & $26(9.8)$ & & \\
\hline \multicolumn{5}{|c|}{ Joint effusion at clinical examination§ } \\
\hline Moderate-important & $153(75.0)$ & $51(25.0)$ & $<0.0001$ & $1.97(1.19 ; 3.23)$ \\
\hline \multirow[t]{2}{*}{ Absent-minimal } & $347(87.6)$ & 49 (12.4) & & \\
\hline & \multicolumn{4}{|c|}{ US joint effusion } \\
\hline \multicolumn{5}{|l|}{ Sex } \\
\hline Female & $262(60.4)$ & $172(39.6)$ & 0.0016 & $0.62(0.41$ to 0.94$)$ \\
\hline Male & $76(46.1)$ & $89(53.9)$ & & \\
\hline \multicolumn{5}{|l|}{ Radiological K\&L grades } \\
\hline $3-4$ & $162(48.6)$ & $171(51.4)$ & $<0.0001$ & 1.91 (1.32 to 2.77$)$ \\
\hline $0-2$ & $176(66.2)$ & $90(33.8)$ & & \\
\hline \multicolumn{5}{|c|}{ Joint effusion at clinical examination§ } \\
\hline Moderate-important & $93(45.6)$ & $111(54.4)$ & 0.0001 & 2.70 (1.76 to 4.16$)$ \\
\hline Absent-minimal & $245(62.0)$ & $150(38.0)$ & & \\
\hline \multicolumn{5}{|c|}{ Sudden aggravation of knee pain ${ }^{* *}$} \\
\hline Severe-very severe & $143(52.0)$ & $132(48.0)$ & 0.0441 & $1.77(1.16$ to 2.71$)$ \\
\hline Absent-minimal-moderate & $195(60.2)$ & $129(39.8)$ & & \\
\hline
\end{tabular}

*p Value of univariate analysis; $\mathrm{OOR}$ : odds ratio, $95 \% \mathrm{Cl}$ : $95 \%$ confidence interval, OR and $95 \% \mathrm{Cl}$ are adjusted though a logistic regression model analysing the probability of the presence of knee synovitis or joint effusion at US; $\ddagger K \& L$, Kellgren and Lawrence radiological grade $\geqslant 3$, or $<3$ observed in both femorotibial compartments of the studied knee; §joint effusion: absent or minimal versus moderate or important; " sex: female versus male; **sudden aggravation of knee pain in the previous 2 weeks: absent, minimal or moderate versus severe or very severe. 


\section{Multivariate analysis Synovitis}

We found those subjects with knee OA with a more severe radiological grade (K\&L grade $\geqslant 3$ ) and moderate or important knee joint effusion on clinical examination had an increased probability of synovitis being detected at US examination (odds ratio $(\mathrm{OR})=2.20$ and 1.97, respectively).

\section{Joint effusion}

Subjects with knee OA with a more severe radiological grade (K\&L grade $\geqslant 3$ ), sudden aggravation of pain in the previous 2 weeks, and moderate or important knee joint effusion on clinical examination, had an increased probability of a joint effusion being detected at US examination $(\mathrm{OR}=1.91,2.70$, and 1.77, respectively). Interestingly, women had fewer joint effusions than men $(\mathrm{OR}=0.62)$.

The multivariate analyses for US synovitis and joint effusion showed similar results when adjusted for country. Arbitrarily, adjustment for countries was performed using the United Kingdom as reference estimates. Table 5 presents the adjusted results.

Note that whatever the definition of US inflammation (synovitis or effusion), the results of the multivariate analysis were the same: subjects with knee OA with a more severe radiological grade (K\&L grade $\geqslant 3$ ) and moderate or important knee joint effusion on clinical examination had an increased likelihood of inflammation on US examination. Conversely, those with a lesser degree of radiological damage and no or mild joint effusion on clinical examination had an increased probability of no synovitis or effusion being detected on US examination. A clinical history of no sudden increase in pain intensity in the previous 2 weeks also increased the probability of no detectable US effusion.

A multivariate analysis was then conducted separately in the subgroups of subjects with earlier $(K \& L$ score $<3)$ and more advanced $(K \& L \geqslant 3)$ radiographic disease, respectively, in both femorotibial compartments. In subjects with earlier radiographic stage, the OR of joint effusion at clinical examination was increased to $4.11(p=0.003)$ and 3.42 $(p=0.0002)$ for prediction of US synovitis and joint effusion, respectively; the OR of sudden aggravation of knee pain in the previous 2 weeks increased to $3.26(\mathrm{p}=0.0006)$ for prediction of US joint effusion; sex was no longer predictive for inflammation at US examination.

\section{DISCUSSION}

This was the first large, multicentre, international study to examine the prevalence of synovitis and/or joint effusion as detected by US in subjects with symptomatic, chronic knee OA. The prevalence of US features suggestive of an inflammatory process, either synovitis or effusion, was quite high (47\%). A large group of patients also had US detected effusion and no detectable synovitis $(30 \%)$. These findings may reflect the strict definitions of synovitis and effusion employed in this study; and, possibly, microscopic synovitis may exist in the absence of US detected synovial hypertrophy.

Although it has been shown that US is a useful instrument for assessing joint effusion and synovitis in inflammatory rheumatic diseases, its usefulness in OA has not yet been established, perhaps owing to the limitations of this technique in evaluating cartilage, the focus of much OA research. ${ }^{14-1632}$ Another reason may be the idea that this technique is considered too "operator dependent". To examine this problem, and in the absence of agreed definitions and acquisition protocols for US detected synovial disease, we established conservative definitions (measurement cut off points) for both synovial thickness and joint effusion depth; we only measured synovial disease in the suprapatellar compartment of the knee and used grey scale US for defining synovitis (instead of Doppler techniques, which have greater variability between machines and readers). A training session was also used to improve reliability across the large numbers of centres.

Our results suggest that US inflammation was not detected in at least half the subjects with chronic, symptomatic knee OA. We found no correlations between US inflammatory signs and pain intensity during recent physical activity. One explanation might be that our study focused on the assessment of synovial features alone in painful knee OA and we did not explore other possible sources of pain. Certainly this supports recent MRI studies, which suggest that non-synovial features such as bone marrow lesions may be associated with pain. ${ }^{33}$

Clinical features were not good predictors of US synovial disease-even in the third of subjects with two out of four clinical features considered suggestive of inflammation, only half had inflammation confirmed at US examination. Features such as night pain and morning stiffness $>30$ minutes did not correlate with synovitis or joint effusion.

It has been suggested that synovitis and joint effusion are secondary phenomena in OA as a consequence of chondrolysis; and that the inflammatory process, and in particular the synthesis of matrix degrading enzymes, subsequently aggravates cartilage breakdown, resulting in an amplifying cycle and perhaps explaining the more rapid progression of chondropathy in late stage disease. ${ }^{34}$ Certainly this study confirmed the high correlation between the radiographic stage of $\mathrm{OA}$ and the inflammatory process. The presence of inflammatory signs observed at US was statistically associated with advanced radiographic disease as expressed by high radiological scores (K\&L grade 3-4), and at an earlier stage (K\&L grade 1-2) with clinical signs or symptoms suggestive of an inflammatory flare-for example, severe or very severe sudden aggravation of pain in the previous 2 weeks, or moderate or marked joint effusion. Note that this subgroup analysis was exploratory and no definite conclusions can be drawn owing to the small number of subjects. Conversely, for subjects with painful knee OA and less severe radiological grade $(K \& L$ grade $<3)$, and with no/minimal knee joint effusion at clinical examination and sudden aggravation of pain in the previous 2 weeks, the likelihood of no US inflammatory features was increased. Moreover, the use of any drugs was not related to US synovitis or effusion.

There are some limitations to this study. Firstly, some heterogeneity was found between countries for most of the characteristics at clinical examination. This may reflect differences in healthcare systems and thus subject referral. Also, the distribution of synovial thickness and effusion depth was not normal, probably owing to a technical limitation in measuring very small amounts of synovial tissue or effusion. As mentioned, in order to reduce interobserver variability, we had a training session before the study started. However, no formal assessment of interobserver reliability was made owing to the large number of ultrasonographers taking part in the study (as commonly occurs with other studies which use a large number of examiners performing, for example, swollen joint counts). Clearly, additional studies in this field need to be performed to determine standardised definitions and acquisition based on normal and arthritic cohorts; some of these problems are currently being examined by a EULAR/OMERACT US working group.

This study demonstrated that US detected synovitis and effusion are common in painful knee OA and confirmed previous reports of the lack of sensitivity of clinical features in predicting inflammation of the synovium. In particular, this study clearly emphasises that synovitis is more commonly 
seen in advanced radiographic disease than in early radiographic disease, suggesting that US may be more useful for detecting inflammation in early OA. Further studies are required in order to better understand the underlying process driving OA synovitis and whether this process differs in early and late disease.

\section{ACKNOWLEDGEMENTS}

We gratefully acknowledge the contribution of all investigators. France: Dr Yves Alibert, Dr Xavier Ayral, Dr Alain Beaulieu-Camus Dr Albert Benbassat, Dr Jean-Michel Benoit, Dr Pierre Borderie, Professor Pierre Bourgeois, Dr Jacques Busson, Dr Alain Cottin, Dr Catherine Cyteval, Dr Catherine Didry, Dr Jean Durckel, Dr Fabien Etchepare, Dr Véronique Ferrazzi, Dr Dominique Folinais, Dr Denis Fornecker, Dr Philippe Gibeault, Dr Eric Gibert, Dr Christophe Hudry, Dr Dominique Kieffer, Dr Marie-Christine Legouffe, Dr Emmanuel Maheu, Dr Philippe Mathieu, Dr Frédéric Maury, Dr Francis Meyer, Dr Gérard Mirisch, Dr Gérard Morvan, Dr Jean-François Moussali, Dr Minh Nguyen, Dr Gérard Panis, Dr Eric Pansard, Dr Stephan Pavy, Dr Sylvie Rozenberg, Dr Christine Saudeau, Dr Bruno Singer, Dr Marc Tardieu, Dr Philippe Tauveron, Dr Philippe Thelen, Dr Roland Vaisberg, Dr Marc Wybier.

Italy: Dr Patrizia Blasetti, Dr Rossella De Angelis, Dr Antonella Farina, Dr Emilio Filippucci, Dr Gianni Lamanna.

Spain: Dr Gema Bonilla, Dr Eugenio De Miguel.

Germany: Dr Eugen Feist, Dr Sonja Kary, Dr Uwe Lang, Dr Anett Reissauer, Dr Udo Schneider.

Switzerland: Professeur Jean-Charles Gerster, Dr Laurent Malterre, Dr Georges Rappoport, Dr Nicholas Theumann, Dr Daniel Van Linthoudt, Dr Maurice Waldburger, Dr Pascal Zufferey.

Belgium: Dr Béatrice Andre, Dr Marie-Joëlle Kaiser, Dr Olivier Kaye, Dr Stefaan Marcelis.

This study was supported in part by an unrestricted grant from Bristol Myers-Squibb.

\section{Authors' affiliations}

M A D'Agostino*, M Dougados, Rheumatology Department, Cochin Hospital, Paris, France

P Conaghan, R Wakefield, P Emery, University of Leeds and Rheumatology Department, Leeds General Infirmary, Leeds, UK M Le Bars, N Schmidely, Bristol Myers-Squibb, Reuil-Malmaison, France G Baron, P Ravaud, Epidemiology, Biostatistics and Clinical Research Department, Bichat Hospital, Paris, France

W Grassi, Rheumatology Department, Jesi Hospital, Jesi, Italy E Martin-Mola, Rheumatology Department, La Paz Hospital, Madrid, Spain

J-L Brasseur, Radiology Department, Pitié Salpêtrière Hospital, Paris, France

A So, Rheumatology Department, Vaudois Hospital, Lausanne, Switzerland

M Backhaus, G Burmester, Rheumatology Department, Charité University Hospital, Berlin, Germany

M Malaise, Rheumatology Department, Saint Tiltman Hospital, Liege, Belgium

*Current address: Rheumatology Department, Ambroise Paré Hospital, Boulogne-Billancourt, France.

\section{REFERENCES}

1 Creamer P, Hunt M, Dieppe P. Pain mechanisms in osteoarthritis of the knee: effect of intra-articular anaesthetic. J Rheumatol 1996;23:1031-6.

2 Likar R, Schafer M, Paulak F, Sitt R, Pipam W, Schalk H, et al. Intra-articular morphine analgesia in chronic pain subjects with osteoarthritis. Anesth Analg 1997;84:1313-17.

3 Amor B. Congestive outbreak of osteoarthritis: chondrolysis and cartilage repair. Rev Prat 1993;43:601-3.

4 Dougados M. Clinical assessment of osteoarthritis in clinical trials. Curt Opin Rheumatol 1995;7:87-91.

5 GREES. Group for the Respect of Ethics and Excellence in Science: osteoarthritis section. Recommendations for the registration of drugs used in the treatment of osteoarthritis. Ann Rheum Dis 1996;55:552-7.
6 Bellamy N, Kirwan J, Altman R, Boers M, Brandt K, Brooks P, et al. Recommendations for a core set of outcome measures for future phase III clinical trials in knee, hip and hand osteoarthritis. Results of consensus development at OMERACT III. J Rheumatol 1997;24:799-802.

7 Ayral X, Pickering EH, Woodworth TG, Mackillop N, Dougados M. Synovitis predicts the arthroscopic progression of medial tibiofemoral knee osteoarthritis [abstract]. Arthritis Rheum 2001;44(suppl 9):S101.

8 Dieppe P, Cushnaghan J, Young P, Kirwan J. Prediction of the progression of joint space narrowing of the knee by bone scintigraphy. Ann Rheum Dis 1993;52:557-63.

9 Dougados M, Gueguen A, Nguyen M, Thiesce A, Listrat V, Jacob L, et al. Longitudinal radiologic evaluation of osteoarthritis of the knee. J Rheumatol 1992;19:378-84

10 Ayral X, Ravaud P, Bonvarlet JP, Simonnet J, Lecurieux R, Nguyen M, et al. Arthroscopic evaluation of post-traumatic patellofemoral chondropathy. J Rheumatol 1999;26:1140-7.

11 Fernandez-Madrid F, Karvonen RL, Teitge RA, Miller PR, An T, Negendank WG. Synovial thickening detected by magnetic resonance imaging in osteoarthritis of the knee confirmed by biopsy as synovitis. Magn Reson Imaging 1995;2:177-83.

12 Hill CL, Gale DG, Chaisson CE, Skinner K, Kazis L, Gale E, et al: Knee effusion, popliteal cysts, and synovial thickening: association with knee pain in osteoarthritis. J Rheumatol 2001;28:1330-7.

13 Hammer M, Mielke H, Wagener P, Schwarzrock R, Giebel G. Sonography and NMR imaging in rheumatoid gonarthritis. Scand J Rheumatol 1986; 15:157-64.

14 Walther M, Harms H, Krenn V, Radke S, Faendrich TP, Gohlke F. Correlation of power Doppler sonography (PDS) in the diagnosis of synovial hypertrophy of the knee joint by verifying and comparing the PDS findings with histopathologic findings of synovial membrane vascularity. Arthritis Rheum 2001;44:331-8.

15 Aisen AM, McCune WJ, MacGuire A, Carson PL, Silver TM, Jafri SZ, et al. Sonographic study of the cartilage of the knee. Radiology 1984;153:781-4.

16 Grassi W, Lamanna G, Cervini C. Sonographic imaging of normal and osteoarthritic cartilage. Semin Arthritis Rheum 1999;28:398-403.

17 Kane D, Balint PV, Sturrock RD. Ultrasonography is superior to clinical examination in the detection and localization of knee joint effusion in rheumatoid arthritis. J Rheumatol 2003;30:966-71.

18 Fiocco U, Cozzi L, Rubaltelli L, Rigon C, De Candia A, Tregnaghi A, et al. Long-term sonographic follow-up of rheumatoid and psoriatic proliferative knee joint synovitis. Br J Rheumatol 1996;35:155-63.

19 Fornage B. Muskoloskeletal ultrasound. New York: Churchill Livingstone, 1995:201-9.

20 Altman R, Asch E, Bloch D, Bole G, Borenstein K, Brandt K, et al. Development of criteria for the classification and reporting of osteoarthritis. Classification of osteoarthritis of the knee. Arthritis Rheum 1986;29:1039-49.

21 Kellgren JH, Lawrence JS. Radiological assessment of osteoarthrosis. Ann Rheum Dis 1957;16:494-502.

22 Kellgren JH, Jeffrey MR, Ball J. The epidemiology of chronic rheumatism: atlas of standard radiographs, Vol 2. Oxford: Blackwell Scientific, 1963.

23 Steinbrocker O, Traeger CH, Batterman RC. Therapeutic criteria in rheumatoid arthritis. JAMA 1949;140:659-62.

24 Wakefield RJ, Brown AK, O'Connor PJ, Emery P. Power Doppler sonography: improving disease activity assessment in inflammatory musculoskeletal disease. Arthritis Rheum 2003;48:285-8.

25 Rubaltelli L, Fiocco U, Cozzi L. Prospective sonographic evaluation of proliferative knee joint synovitis. J Ultrasound Med 1992;13:855-62.

26 Backhaus M, Burmester GR, Gerber T, Grassi W, Machold KP, Swen A, et al. Guidelines for musculoskeletal ultrasound in rheumatology. Ann Rheum Dis 2001;60:641-9.

27 Dougados M. La mesure: méthodes d'évaluation des affections rhumatismales. Paris: Expansion Scientifique, 1997.

28 Bellamy N, Buchanan WW, Goldsmith CH, Campbell J, Stitt LWJ. Validation of WOMAC: a health status instrument for measuring clinically important patient relevant outcomes to antirheumatic drug therapy in subjects with osteoarthritis of the hip or knee. J Rheumatol 1988;15:1833-40.

29 Dougados M, Nguyen M, Caporal R, Legeais J, Bouxin-Sauzet A, PellegriGuegnault B, et al. Ximoprofen in ankylosing spondylitis. A double blind placebo controlled dose ranging study. Scand J Rheumatol 1994;23:243-8.

30 Dougados M, Nguyen M, Nakache JP, Amor B. Spondylarthrite ankylosante et anti-inflammatoires. Concours Med 1987;30:2780-6.

31 Buderer NM. Statistical methodology. Incorporating the prevalence of disease into the sample site calculation for sensitivity and specificity. Acad Emerg Med 1996;3:824

32 Karim Z, Wakefield RJ, Quinn PG, Conaghan P, Brown AK, Veale P, et al. Validation and reproducibility of ultrasonography in the detection of synovitis in the knee. A comparison with arthroscopic and clinical examination. Arthritis Rheum 2004;2:387-94.

33 Felson DT, Chaisson CE, Hill CL, Totterman SM, Gale ME, Skinner KM, et al. The association of bone marrow lesions with pain in knee osteoarthritis. Ann Intern Med, 2001;3, 134:541-9.

34 Pelletier JP, Martel-Pelletier J, Abramson SB. Osteoarthritis, an inflammatory disease. potential implication for the selection of new therapeutic targets. Arthritis Rheum 2001;44:1237-47. 